\title{
Influence of the CYP1A1 T3801C Polymorphism on Tobacco and Alcohol-Associated Head and Neck Cancer Susceptibility in Northeast India
}

\author{
Seram Anil Singh ${ }^{\star}$, Javed Hussain Choudhury ${ }^{\star}$, Wetetsho Kapfo, Sharbadeb \\ Kundu, Bishal Dhar, Shaheen Laskar, Raima Das, Manish Kumar, Sankar \\ Kumar Ghosh*
}

\begin{abstract}
Background: Tobacco and alcohol contain or may generate carcinogenic compounds related to cancers. CYP1A1 enzymes act upon these carcinogens before elimination from the body. The aim of this study was to investigate whether CYP1A1 T3801C polymorphism modulates the relationship between tobacco and alcoholassociated head and neck cancer (HNC) susceptibility among the northeast Indian population. Materials and Methods: One hundred and seventy histologically confirmed HNC cases and 230 controls were included within the study. The CYP1A1 T3801C polymorphism was determined using PCR-RFLP, and the results were confirmed by DNA sequencing. Logistic regression (LR) and multifactor dimensionality reduction (MDR) approaches were applied for statistical analysis. Results: The CYP1A1 CC genotype was significantly associated with HNC risk $(\mathrm{P}=0.045)$. A significantly increased risk of $\mathrm{HNC}(\mathrm{OR}=6.09 ; \mathrm{P}<0.0001)$ was observed in individuals with combined habits of smoking, alcohol drinking and tobacco-betel quid chewing. Further, gene-environment interactions revealed enhanced risks of $\mathrm{HNC}$ among smokers, alcohol drinkers and tobacco-betel quid chewers carrying CYP1A1 TC or CC genotypes. The highest risk of $\mathrm{HNC}$ was observed among smokers $(\mathrm{OR}=7.55 ; \mathrm{P}=0.009)$ and chewers $(\mathrm{OR}=10.8 ; \mathrm{P}<0.0001)$ carrying the CYP1A1 CC genotype. In MDR analysis, the best model for HNC risk was the three-factor model combination of smoking, tobacco-betel quid chewing and the CYP1A1 variant genotype $(\mathrm{CVC}=99 / 100 ; \mathrm{TBA}=0.605 ; \mathrm{P}<0.0001)$; whereas interaction entropy graphs showed synergistic interaction between tobacco habits and CYP1A1. Conclusions: Our results confirm that the CYP1A1 T3801C polymorphism modifies the risk of $\mathrm{HNC}$ and further demonstrated importance of gene-environment interaction.
\end{abstract}

Keywords: Head and neck cancer - CYP1A1 T3801C polymorphism - tobacco - alcohol - northeast India

Asian Pac J Cancer Prev, 16 (16), 6953-6961

\section{Introduction}

Head and neck cancers (HNC) are a heterogeneous group of cancers that include cancers of the skin of the head and neck, nasal cavity, paranasal sinuses, nasopharynx, lip, oral cavity, oropharynx, larynx, hypopharynx, trachea, neck, salivary glands and the parapharyngeal region (Siegel et al., 2014). Incidence of HNC has increased at an alarming rate for the past 10 years. It is the fifth most prevalent among all cancers throughout the world with estimated death of 355 per 100,000 (Masood et al., 2014). These cancers occur more frequently in men (male: female ratio of 4-5:1). In India, HNC acquires 30-40\% cancers of all sites and is the sixth most common cause of death in males and seventh in females (Bhattacharjee et al., 2006).

Tobacco smoking and betel quid chewing with or without tobacco are major risk factors to be associated with HNC (Mondal et al., 2013; Amtha et al., 2014;
Choudhury et al., 2014; Choudhury and Ghosh, 2014). Alcohol habit is another risk factor associated with HNC (Risch et al., 2003; Hashibe et al., 2009; Ghosh et al., 2014b). The magnitude of risk conferred by the interaction between tobacco, and alcohol is still unclear. Studies have implicated that many carcinogens present in tobacco, and alcohols are metabolized to active forms that have deleterious effects in our body. These activated substances can cause oxidative reactions in tissues, and initiate reactions to produce free radicals (Cury et al., 2012). The presence of reactive oxygen species (ROS) can cause damage to cellular biomolecules, including protein and DNA, consequently resulting in carcinogenesis (Mondal and Ghosh, 2013; Kumar and Muniyandi, 2015).

Metabolic enzymes that are potentially involved in either the activation (phase I) or detoxication (phase II) of chemical carcinogens have received a great deal of attention recently as possible genetic susceptibility factors 
for a variety of cancers (Olshan et al., 2000). Human cytochrome $\mathrm{P} 450$, family 1 , subfamily $\mathrm{A}$, polypeptide 1 (CYP1A1) is one of the major phase I enzymes, involved in the activation of carcinogens related to head and neck cancers, such as polycyclic aromatic hydrocarbon (PAH), heterocyclic amines, N-nitrosamine, alkaloids, polyphenols and tannins present in tobacco smoke and betel quid (Wu et al., 2004; Sharan et al., 2012). Ethanol and acetaldehyde of alcoholic drinks are important chemical agents related to various cancers (Lachenmeier et al., 2012). Therefore, modulation of CYPIAl activity may be important in the aetiology of HNC. Both environmental and genetic factors influence the activity of CYPIAl. Tobacco smoking and betel quid consumption increases $C Y P 1 A 1$ activity in humans. $C Y P 1 A 1$ activity is also modulated by specific polymorphisms in the CYP1Al gene. One of its common polymorphisms CYP1Al T3801C (CYP1A1-MspI or CYP1Al*2A or rs4646903) has been associated with higher induction of $C Y P 1 A 1$. The higher enzyme activity would result in increased levels of carcinogenic intermediates, leading to greater risk of cancer development. The associations of these CYPIAl single nucleotide polymorphisms (SNPs) with cancers have been well documented (Jiang et al., 2014; Liu et al., 2014; Lu et al., 2014).

The aim of this study was to investigate the frequency of CYP1A1 T3801C polymorphism in patients with HNC and its role in modulating the relationship between tobacco and alcohol consumption, and HNC risk. Furthermore, multifactor dimensionality reduction (MDR) approach was used to investigate the high-degree gene-environmental interaction in NPC carcinogenesis.

\section{Materials and Methods}

\section{Study population}

The present study consists of 170 histologically confirmed, untreated HNC cases (diagnosed between December, 2009 and July, 2013) and 230 healthy controls (without family history of cancer). The oral swab and/ or peripheral blood of the participating subjects were collected upon written consent. Controls were individually matched to cases in sex, age, ethnicity and neighborhood. Further, a pre-designed questionnaire was used to collect demographic information including family history of cancer, habits of tobacco, and alcohol consumption as well as their frequencies. Tobacco habits in our study include smoking (cigarettes and beedi), tobacco chewing (khaini, zarda, gutkha), and betel quid chewing (betel leaf along with areca nut, lime with or without tobacco). The study was approved by the Institutional Ethics Committee, Assam University, Silchar. Precautions were taken to avoid contamination while collecting and processing the samples.

\section{DNA extraction and genotyping}

Genomic DNA was isolated from the collected blood or oral swabs by phenol/chloroform/ isoamylalcohol (Ghosh and Mondal, 2012) method, and then stored in T.E buffer (10Mm Tris-HCl, pH 0.8, $1 \mathrm{Mm}$ EDTA) at $-20^{\circ} \mathrm{C}$ for further used. The polymorphisms of CYP1A1 T3801C gene was analysed by polymerase chain reaction restriction length polymorphism (PCR-RFLP) method ( $\mathrm{Ng}$ et al., 2005). PCR amplification of a 343-base DNA fragment containing MspI restriction site was performed, using the primers 5/-TAGGAGTCTTGTCTCATGCCTT-3/ and 5/-CAGTGAAGAGGTGTAGCCGCT-3/. PCR programme was performed at $95^{\circ} \mathrm{C}$ for $5 \mathrm{~min}$ for the initial denaturation, following 30 cycles of denaturation at $95^{\circ} \mathrm{C}$ for $30 \mathrm{~s}$, annealing at $62^{\circ} \mathrm{C}$ for $45 \mathrm{~s}$, extension at $72^{\circ} \mathrm{C}$ for $30 \mathrm{~s}$ and final extension at $72^{\circ} \mathrm{C}$ for 5 mins. For the CYP1A1 T3801C genotype analysis, the PCR product was digested with the MspI restriction enzyme (New England BioLabs, USA); a single 343 bp fragment represents the wild-type allele (TT), three fragments of 343, 200 and 143 bp indicates for the heterozygous (TC) and two fragments of 200 and $143 \mathrm{bp}$ for the variant allele (CC). The RFLP results were confirmed by sequencing $10 \%$ of randomly selected samples from both cases and controls by Sanger sequencing using Genetic Analyzer 3500, Applied BioSystems (Molecular Medicine Lab, Department of Biotechnology, Assam University, Silchar, India).

\section{Statistical analysis}

Statistically significant differences between cases and controls for demographic characteristics were assessed by $\chi^{2}$ test. The association between smoking, alcohol drinking and tobacco-betel quid chewing and CYP1Al T3801C polymorphism in $\mathrm{HNC}$ risk were analyzed by calculating odds ratios (ORs), $95 \%$ confidence intervals $(95 \% \mathrm{CI}$ ), and their corresponding P-values. A P-value of $<0.05$ were considered to indicate statistically significant. Departures from Hardy-Weinberg equilibrium for CYP1A1 T3801C genotype were evaluated by comparing the observed genotype frequencies with expected frequencies using $\chi^{2}$ tests. Statistical analyses were performed using SPPS software, version 16 for Windows.

\section{Multifactorial dimensionality reduction analysis}

The MDR analysis is an advanced non-parametric approach (www. multifactordimensionalityreduction. org) used to detect the gene-environment interactions associated with disease risk (Hahn et al., 2003). The advantage of using MDR is it overcomes the sample size limitations often encountered by parametric approaches (example: logistic regression analysis) while studying high-level interaction. The best prediction model was selected on the basis of maximum training balance accuracy (TrBA), testing balance accuracy (TBA) and cross validation consistency (CVC). The best model with highest TrBA, TBA and CVC was tested by 1000 fold permutation testing and $\chi^{2}$ test at 0.05 significance levels during MDR analysis.

\section{Interaction entropy graphs}

The interaction entropy graphs were constructed using MDR results to determine synergistic and nonsynergistic interactions among the variables (Lavender et al., 2009; Yu et al., 2014). The graphs comprise of nodes containing percentage entropy of each individual variables and connections joining them pairwise showing entropy of interaction between them. Positive entropy signifies 
CYP1A1 T3801C Polymorphism and Tobacco and Alcohol-Associated Head and Neck Cancer Susceptibility in Northeast India

synergy and negative entropy indicate redundancy, whereas, zero entropy indicates independence.

\section{Results}

Relationship between tobacco habits and alcohol drinking and the risk for $\mathrm{HNC}$

The present study includes 170 cases and 230 controls. There were no significant differences between cases and controls in terms of gender and age. $67.6 \%$ cases and $60.9 \%$ controls were male; $44.7 \%$ cases and $47.4 \%$ controls were $\leq 50$ years of age $(\mathrm{P}=0.1976$ and 0.6629 , respectively). Smokers were significantly higher among cases than controls $(\mathrm{P}<0.001)$. Differences in tobacco-betel quid chewing and alcohol intake status was observed between cases and controls $(\mathrm{P}<0.05)$. Of the 170 cancer patients, $63(37.1 \%)$ had oral, 60 $(35.3 \%)$ had nasopharyngeal, $24(14.1 \%)$ had laryngeal, $10(5.9 \%)$ had pharyngeal, and $13(7.6 \%)$ had cancer in other head and neck region. We analyzed HNC risk in relation to smoking, alcohol drinking and tobacco-betel quid chewing, with adjustment for possible confounding factors (Table 1). Tobacco habits showed a dose-dependent increased risk of HNC. Heavy smokers $(\mathrm{OR}=2.9,95 \%$ CI: 1.68-4.99; $\mathrm{P}<0.0001)$ and tobacco-betel quid chewers $(\mathrm{OR}=3.0,95 \% \mathrm{CI}: 1.8-5.03 ; \mathrm{P}<0.0001)$ had significantly increased risk of HNC. Similarly, heavy alcohol drinkers had nearly 2 fold (95\% CI: $1.07-3.15 ; \mathrm{P}=0.025)$ increased risk of HNC. The risk of HNC increased 5 fold $(95 \% \mathrm{CI}$ : 2.53-10.0; $\mathrm{P}<0.0001)$ for smokers with tobacco-betel quid chewing. Similarly, significantly increased risks of HNC were observed for alcohol drinkers with smoking habit $(\mathrm{OR}=3.32,95 \% \mathrm{CI}: 1.77-6.24 ; \mathrm{P}<0.0001)$ or with tobacco betel quid chewing (OR=4.18,95\% CI: 2.09-8.33; $\mathrm{P}<0.0001)$. However, highest risk of $\mathrm{HNC}(\mathrm{OR}=6.09,95 \%$ CI: 2.35-15.73; $\mathrm{P}<0.0001)$ was observed in individuals with combine habits of smoking, alcohol drinking and tobacco-betel quid chewing (Table 1).

\section{Polymorphism in CYPlAl gene and association with} HNC risk

The genotypes of CYP1A1 T3801C were determined by detecting the PCR-RFLP band pattern on $1.5 \%$ agarose gel. The PCR-RFLP result was confirmed by randomly sequencing $10 \%$ of the samples (Figure 1). The three genotypes of CYP1A1 T3801C viz. TT, TC and CC had frequency distributions of $45.3 \%, 41.2 \%, 13.5 \%$ and $54.3 \%, 36.1 \%, 9.6 \%$ in cases and controls, respectively. Logistic regression analysis show that CYP1Al CC $(\mathrm{OR}=2.06,95 \% \mathrm{CI}: 1.01-4.17 ; \mathrm{P}=0.045)$ genotype had significant risk association with $\mathrm{HNC}$ in the study population when compare to the TT genotype (Table 2). Combine TC and CC (TT + CC) genotypes also showed a risk $(\mathrm{OR}=1.54,95 \% \mathrm{CI}: 1.02-2.39, \mathrm{P}=0.039)$ of $\mathrm{HNC}$. The risk associated with each allele was also investigated. We observed significant risk of $\mathrm{HNC}$ in those individual

\section{Table 1. Environmental Risk Factors and Risk of Head And Neck Squamous Cell Carcinoma}

\begin{tabular}{|c|c|c|c|c|}
\hline Risk factors & $\begin{array}{c}\text { Cases } \\
\mathrm{n}=170(\%)\end{array}$ & $\begin{array}{c}\text { Controls } \\
\mathrm{n}=230(\%)\end{array}$ & $\mathrm{OR} *(95 \% \mathrm{CI})$ & P-value \\
\hline \multicolumn{5}{|l|}{ Smoking } \\
\hline Never & $65(38.2)$ & $131(57)$ & 1 (ref) & \\
\hline Light & $45(26.5)$ & $58(25.2)$ & $1.58(0.92-2.7)$ & 0.094 \\
\hline Heavy & $60(35.3)$ & $41(17.8)$ & $2.9(1.68-4.99)$ & $<0.0001$ \\
\hline \multicolumn{5}{|c|}{ Tobacco-betel quid chewing } \\
\hline Never & $47(27.6)$ & $102(44.3)$ & 1 (ref) & \\
\hline Light & $51(30)$ & $75(32.6)$ & $1.49(0.89-2.49)$ & 0.126 \\
\hline Heavy & $72(42.4)$ & $53(23)$ & $3.0(1.8-5.03)$ & $<0.0001$ \\
\hline \multicolumn{5}{|c|}{ Alcohol drinking } \\
\hline Never & $74(43.5)$ & $125(54.3)$ & 1 (ref) & \\
\hline Light & $52(30.6)$ & $60(26.1)$ & $1.6(0.97-2.64)$ & 0.064 \\
\hline Heavy & $44(25.9)$ & $45(19.6)$ & $1.84(1.07-3.14)$ & 0.025 \\
\hline \multicolumn{5}{|c|}{ Smoking + Tobacco-betel quid chewing } \\
\hline Never & $17(10)$ & $56(24.3)$ & 1 (ref) & \\
\hline Both & $77(45.3)$ & $52(22.6)$ & $5.04(2.53-10.02)$ & $<0.0001$ \\
\hline Single & $76(44.7)$ & $122(53)$ & $2.09(1.11-3.94)$ & 0.022 \\
\hline \multicolumn{5}{|c|}{ Alcohol drinking + Smoking } \\
\hline Never & $27(15.9)$ & $70(30.4)$ & 1 (ref) & \\
\hline Both & $55(32.4)$ & $44(19.1)$ & $3.32(1.77-6.24)$ & $<0.0001$ \\
\hline Single & $88(51.8)$ & $116(50.4)$ & $1.79(1.04-3.09)$ & 0.034 \\
\hline \multicolumn{5}{|c|}{ Alcohol drinking + Tobacco-betel quid chewing } \\
\hline Never & $15(8.8)$ & $57(24.8)$ & 1 (ref) & \\
\hline Both & $65(38.2)$ & $54(23.5)$ & $4.18(2.09-8.33)$ & $<0.0001$ \\
\hline Single & $90(52.9)$ & $119(51.7)$ & $2.69(1.4-5.15)$ & 0.003 \\
\hline \multicolumn{5}{|c|}{ Smoking + Tobacco-betel quid chewing + Alcohol drinking } \\
\hline Never & $9(5.3)$ & $30(13)$ & 1 (ref) & \\
\hline Both & $39(22.9)$ & $21(9.1)$ & $6.09(2.35-15.73)$ & $<0.0001$ \\
\hline Multiple & $122(71.8)$ & $179(77.8)$ & $2.26(1.01-5.05)$ & 0.047 \\
\hline
\end{tabular}

*Odds ratio $(\mathrm{OR})$ are adjusted for age, gender, smoking, and alcohol drinking and tobacco-betel quid chewing as appropriate; $\mathbf{P}<\mathbf{0 . 0 5}$ considered as statistically significance; Bold values indicate statistical significance $(\mathbf{P}<\mathbf{0 . 0 5})$ 
Seram Anil Singh et al

Table 2. Distribution of CYP1A1 (T3801C) Genotype in Different Types of Head and Neck Cancer

\begin{tabular}{|c|c|c|c|c|c|c|c|c|c|c|}
\hline \multirow{3}{*}{ Genotype } & \multicolumn{2}{|c|}{ Overall } & \multicolumn{2}{|r|}{ Oral } & \multicolumn{2}{|c|}{ Nasopharyngeal } & \multicolumn{2}{|c|}{ Larynx } & \multicolumn{2}{|c|}{ Pharynx and others } \\
\hline & $\mathrm{Ca} / \mathrm{Co}$ & $\begin{array}{c}\mathrm{OR}^{*} \\
(95 \% \mathrm{CI})\end{array}$ & \multirow[t]{2}{*}{$\mathrm{N}=63$} & $\begin{array}{c}\mathrm{OR}^{*} \\
(95 \% \mathrm{CI})\end{array}$ & \multirow[t]{2}{*}{$\mathrm{N}=60$} & $\begin{array}{c}\mathrm{OR}^{*} \\
(95 \% \mathrm{CI})\end{array}$ & \multirow[t]{2}{*}{$\mathrm{N}=24$} & $\begin{array}{c}\mathrm{OR}^{*} \\
(95 \% \mathrm{CI})\end{array}$ & \multirow[t]{2}{*}{$\mathrm{N}=23$} & $\begin{array}{c}\mathrm{OR}^{*} \\
(95 \% \mathrm{CI})\end{array}$ \\
\hline & $170 / 230$ & P value & & P value & & $P$ value & & $P$ value & & P value \\
\hline TT & $77 / 125$ & 1(ref) & 26 & 1(ref) & 26 & 1(ref) & 9 & 1(ref) & 12 & 1(ref) \\
\hline \multirow[t]{2}{*}{$\mathrm{TC}$} & \multirow[t]{2}{*}{$70 / 83$} & $\begin{array}{c}1.45 \\
(0.92-2.27)\end{array}$ & \multirow[t]{2}{*}{25} & $\begin{array}{c}1.5 \\
(0.79-2.87)\end{array}$ & \multirow[t]{2}{*}{26} & $\begin{array}{c}1.63 \\
(0.84-3.15)\end{array}$ & \multirow[t]{2}{*}{12} & $\begin{array}{c}1.9 \\
(0.72-5.05)\end{array}$ & \multirow[t]{2}{*}{8} & $\begin{array}{c}0.99 \\
(0.37-2.62)\end{array}$ \\
\hline & & 0.108 & & 0.213 & & 0.145 & & 0.194 & & 0.991 \\
\hline \multirow[t]{2}{*}{$\mathrm{CC}$} & \multirow[t]{2}{*}{$23 / 22$} & $\begin{array}{c}2.06 \\
(1.01-4.17)\end{array}$ & \multirow[t]{2}{*}{12} & $\begin{array}{c}2.99 \\
(1.25-7.16)\end{array}$ & \multirow[t]{2}{*}{8} & $\begin{array}{c}2.23 \\
(0.77-6.48)\end{array}$ & \multirow[t]{2}{*}{3} & $\begin{array}{c}2.36 \\
(0.54-10.25)\end{array}$ & \multirow[t]{2}{*}{3} & $\begin{array}{c}1.79 \\
(0.43-7.36)\end{array}$ \\
\hline & & 0.045 & & 0.014 & & 0.138 & & 0.251 & & 0.415 \\
\hline \multirow{2}{*}{$\mathrm{TC}+\mathrm{CC}$} & \multirow[t]{2}{*}{$93 / 105$} & $\begin{array}{c}1.54 \\
(1.02-2.39)\end{array}$ & \multirow[t]{2}{*}{37} & $\begin{array}{c}1.8 \\
(0.99-3.26)\end{array}$ & \multirow[t]{2}{*}{34} & $\begin{array}{c}1.73 \\
(0.92-3.23)\end{array}$ & \multirow[t]{2}{*}{15} & $\begin{array}{c}1.99 \\
(0.79-5.0)\end{array}$ & \multirow[t]{2}{*}{11} & $\begin{array}{c}1.14 \\
(0.47-2.76)\end{array}$ \\
\hline & & 0.039 & & 0.049 & & 0.085 & & 0.141 & & 0.768 \\
\hline T-allele & $224 / 333$ & 1(ref) & 77 & 1(ref) & 78 & 1(ref) & 30 & 1(ref) & 32 & 1(ref) \\
\hline \multirow{2}{*}{$\begin{array}{c}\mathrm{C}- \\
\text { allele } \\
\wedge \wedge\end{array}$} & \multirow[t]{2}{*}{$116 / 127$} & $\begin{array}{c}1.37 \\
(1.01-1.85)\end{array}$ & \multirow[t]{2}{*}{49} & $\begin{array}{c}1.67 \\
(1.11-2.52)\end{array}$ & \multirow[t]{2}{*}{42} & $\begin{array}{c}1.41 \\
(0.92-2.16)\end{array}$ & \multirow[t]{2}{*}{18} & $\begin{array}{c}1.57 \\
(0.85-2.92)\end{array}$ & \multirow[t]{2}{*}{14} & $\begin{array}{c}1.15 \\
(0.59-2.22)\end{array}$ \\
\hline & & 0.044 & & 0.016 & & 0.116 & & 0.178 & & 0.731 \\
\hline
\end{tabular}

*Odds ratio (OR) are adjusted for age, gender, smoking, and alcohol intake and tobacco-betel quid chewing as appropriate; $\mathbf{P}<\mathbf{0 . 0 5}$ considered as statistically significance; ${ }^{\wedge}$ Crude odds ratio (OR); Ca cases, Co controls; Bold values indicate statistical significance $(\mathbf{P}<\mathbf{0 . 0 5})$

(A)

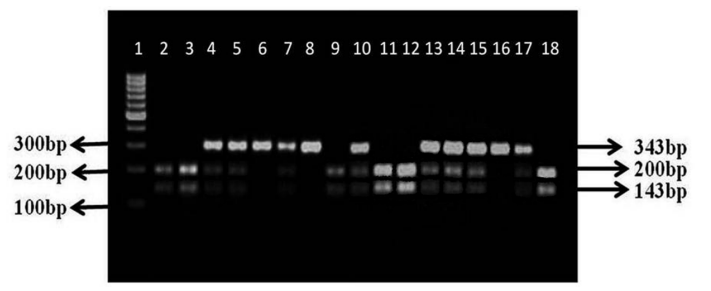

(B)

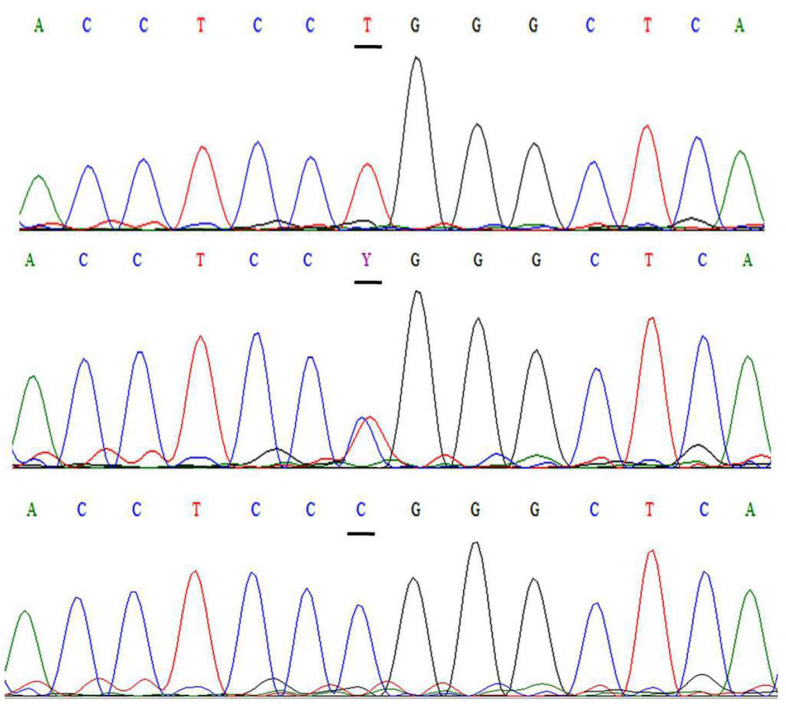

Figure 1.Polymorphism of CYP1A1 T3801C Metabolic

gene. (A) Ethidium bromide stained gel showing CYP1A1 T3801C polymorphism: 100-bp ladder (lane 1); CYP1A1 TT wild genotype (lanes 6, 8 and 16); CYP1A1 TC heterozygous genotype (lanes 4, 5, 7, 10,13,14, 15, and 17); CYP1A1 CC mutant genotype (lanes 2, 3, 9, 11, 12, and 18); (B) DNA sequencing results showing nucleotide changes (mark by black line). Nucleotide change marked as Y indicates the heterozygous genotype, where red peak denotes Thymine $(\mathrm{T})$ while blue peak denotes Cytosine (C) carrying the $\mathrm{C}$-allele as compared to $\mathrm{T}$ - allele $(\mathrm{P}=0.04)$. Further, the association between $C Y P 1 A 1$ T3801C genotype and different types of HNC was analysed. It was observed that $\mathrm{CC}(\mathrm{P}=0.014)$ and combined $\mathrm{TC}+\mathrm{CC}$ $(\mathrm{P}=0.049)$ genotypes were significantly associated with the risk of oral cancer (Table 2). However, CYP1A1 polymorphism was not associated with the risk of other types HNC in our study.

Interaction of tobacco habits and alcohol drinking and CYP1A1 polymorphism in HNC

The potential interactions of smoking, tobacco-betel quid chewing, and alcohol drinking and CYP1A1 T3801C polymorphism in $\mathrm{HNC}$ risk were investigated (Table 3 ). Heavy smokers carrying the CYPlAl TT and TC genotypes had 2.55 fold (95\% CI: 1.27-5.13; $\mathrm{P}=0.012$ ) and 3.42 fold (95\% CI: 1.65-7.11; $\mathrm{P}=0.002)$ increased risk of HNC. However, highest risk was observed among heavy smokers carrying $\mathrm{CC}$ genotype $(\mathrm{OR}=7.55,95 \%$ CI: 1.62-35.1; $\mathrm{P}=0.009)$. We examined interaction of tobacco-betel quid chewing with CYPIAl genotypes in HNC risk. Heavy tobacco-betel quid chewers carrying the TC genotype had 4.91 fold risk (95\% CI: 2.16-11.1; $\mathrm{P}<0.0001)$ of HNC, whereas a 10 fold (95\% CI: 2.09-37.6; $\mathrm{P}<0.0001)$ elevated risk was observed among chewers carrying the CC genotype. Tobacco-betel quid chewing also show significantly interaction with the TT genotype $(\mathrm{P}<0.05)$. Among heavy alcohol drinkers, individuals carrying the TC genotype had significantly increased $(\mathrm{OR}=3.26$, 95\% CI: 1.43-7.43; $\mathrm{P}=0.006)$ risk of $\mathrm{HNC}$, whereas individual with CC genotype had 7.21 fold (95\% CI: $1.55-33.5 ; \mathrm{P}=0.011$ ) elevated risk.

The combinations of smoking, alcohol drinking and tobacco-betel quid chewing habits and their interactions with CYP1A1 T3801C polymorphism in HNC risk were also considered (Table 3 ). A significantly elevated risk of 
CYP1A1 T3801C Polymorphism and Tobacco and Alcohol-Associated Head and Neck Cancer Susceptibility in Northeast India Table 3. Interaction of smoking, alcohol intake and tobacco-betel quid chewing and CYP1A1 polymorphism in HNC

\begin{tabular}{|c|c|c|c|c|c|c|}
\hline \multicolumn{7}{|c|}{ CYP1A1 (T3801C, rs4646903) polymorphism } \\
\hline & \multicolumn{2}{|c|}{ TT genotype } & \multicolumn{2}{|c|}{ TC genotype } & \multicolumn{2}{|c|}{ CC genotype } \\
\hline & \multirow{2}{*}{$\mathrm{Ca} / \mathrm{Co}$} & OR $(95 \% \mathrm{CI})$ & \multirow{2}{*}{$\mathrm{Ca} / \mathrm{Co}$} & $\mathrm{OR}(95 \% \mathrm{CI})$ & \multirow{2}{*}{$\mathrm{Ca} / \mathrm{Co}$} & OR $(95 \% \mathrm{CI})$ \\
\hline & & $* * \mathrm{P}$ value & & $* * \mathrm{P}$ value & & $* * \mathrm{P}$ value \\
\hline \multicolumn{7}{|c|}{ Smoking } \\
\hline \multirow{2}{*}{ Never } & \multirow{2}{*}{$32 / 69$} & \multirow{2}{*}{1 (reference) } & \multirow{2}{*}{$25 / 48$} & $1.12(0.59-2.12)$ & \multirow{2}{*}{$8 / 14$} & $1.23(0.48-3.17)$ \\
\hline & & & & 0.746 & & 0.802 \\
\hline \multirow{2}{*}{ Light } & \multirow{2}{*}{$19 / 34$} & $1.20(0.60-2.41)$ & \multirow{2}{*}{$18 / 18$} & $2.16(1.0-4.65)$ & \multirow{2}{*}{$8 / 6$} & $2.88(0.96-8.65)$ \\
\hline & & 0.719 & & 0.069 & & 0.076 \\
\hline \multirow{2}{*}{ Heavy } & \multirow{2}{*}{$26 / 22$} & $2.55(1.27-5.13)$ & \multirow{2}{*}{$27 / 17$} & $3.42(1.65-7.11)$ & \multirow{2}{*}{$7 / 2$} & $7.55(1.62-35.1)$ \\
\hline & & 0.012 & & 0.002 & & 0.009 \\
\hline \multicolumn{7}{|c|}{ Tobacco-betel quid chewing } \\
\hline \multirow{2}{*}{ Never } & $14 / 55$ & 1 (reference) & $27 / 35$ & $3.03(1.41-6.52)$ & $6 / 12$ & $1.96(0.64-6.0)$ \\
\hline & $14 / 00$ & 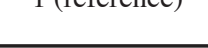 & $2 \pi J$ & 0.005 & 0112 & 0.344 \\
\hline & & $2.59(1.21-5.51)$ & & $2.53(1.11-5.77)$ & & $3.93(1.15-13.4)$ \\
\hline Lignit & 21141 & 0.016 & $10 / 20$ & 0.034 & (0) & 0.063 \\
\hline Heavy & $36 / 29$ & $4.88(2.29-10.4)$ & $25 / 20$ & $4.91(2.16-11.1)$ & $11 / 4$ & $\begin{array}{c}10.80(2.09- \\
37.6)\end{array}$ \\
\hline & & $<0.0001$ & & $<0.0001$ & & $<0.0001$ \\
\hline & & & hol drin & & & \\
\hline Newer & 33168 & 1 (reforence) & $30 / 43$ & $1.44(0.77-2.68)$ & $11 / 4$ & $1.62(0.67-3.89)$ \\
\hline 1980 & (2) & 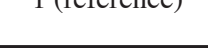 & $50 / 43$ & 0.267 & $11 / 4$ & 0.35 \\
\hline Jiobt & 26126 & $2.06(1.05-4.06)$ & $21 / 28$ & $1.55(0.77-3.10)$ & $5 / 6$ & $1.72(0.51-5.73)$ \\
\hline Lignit & 20120 & 0.053 & $21 / 20$ & 0.277 & J/0 & 0.505 \\
\hline Нооче & $18 / 21$ & $1.20(0.59-2.43)$ & $10 / 12$ & $3.26(1.43-7.43)$ & $7 / 2$ & $7.21(1.55-33.5)$ \\
\hline Heavy & $10 / 01$ & 0.714 & 1312 & 0.006 & IIL & 0.011 \\
\hline & & Smoking & cco-bet & chewing & & \\
\hline Never & $8 / 27$ & 1 (reference) & $6 / 20$ & $1.01(0.31-3.31)$ & $3 / 0$ & $1.13(0.26-4.91)$ \\
\hline Never & $0 / 21$ & I (rererence) & $0 / 20$ & 1 & $3 / 9$ & 1 \\
\hline Sincle & $30 / 70$ & $1.45(0.60-3.51)$ & $30 / 44$ & $2.99(1.23-7.27)$ & & $3.38(0.99-11.5)$ \\
\hline Ning & 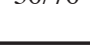 & 0.515 & 列 & 0.023 & $8 / 0$ & 0.102 \\
\hline Roth & $30 / 28$ & $4.70(1.88-11.7)$ & $25 / 10$ & $4.44(1.67-11.7)$ & $12 / 5$ & $8.10(2.26-29)$ \\
\hline DotII & (20 & 0.001 & תו & 0.003 & (2/J & 0.002 \\
\hline & & Alc & inking - & ing & & \\
\hline Neyer & $11 / 30$ & 1 (reference) & $13 / 22$ & $2.10(0.81-5.40)$ & $3 / 0$ & $1.18(0.29-4.87)$ \\
\hline 1rever & $11 / 3$ & (IETerice) & $13 / 22$ & 0.148 & $3 / 9$ & 1 \\
\hline Single & $41 / 50$ & $2.46(1.14-5.33)$ & $32 / 47$ & $2.49(1.12-5.52)$ & $14 / 10$ & $4.96(1.76-13.9)$ \\
\hline Singie & $41 / 59$ & 0.029 & $35 / 4 /$ & 0.035 & $14 / 10$ & 0.003 \\
\hline Both & $25 / 27$ & $3.28(1.4-7.71)$ & $24 / 14$ & $6.08(2.4-15.3)$ & $6 / 3$ & $7.09(1.64-30.6)$ \\
\hline Dour & 年 & 0.007 & $24 / 14$ & $<0.0001$ & $0 / 3$ & 0.013 \\
\hline & & Alcohol drin & Tobacco & quid chewing & & \\
\hline Newer & $5 / 28$ & 1 (reforence) & $24 / 14$ & $2.8(0.80-9.80)$ & $2 / 10$ & $1.12(0.2-6.31)$ \\
\hline Never & गाLO & 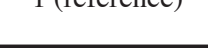 & $24 / 14$ & 0.124 & $2 / 10$ & 1 \\
\hline Single & 37168 & $3.05(1.1-8.44)$ & $40 / 45$ & $4.98(1.78-13.9)$ & $13 / 6$ & $9.60(2.91-35.3)$ \\
\hline Singie & $31 / 00$ & 0.031 & $40 / 4 J$ & 0.001 & $13 / 0$ & 0.001 \\
\hline 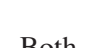 & $35 / 20$ & $6.76(2.35-19.4)$ & 27 & $5.6(1.86-16.8)$ & $81-2>$ & $7.47(1.87-29.8)$ \\
\hline DoII & (3) & $<0.0001$ & $221<2$ & 0.022 & (10 & 0.01 \\
\hline
\end{tabular}

Bold values indicate statistical significance $(\mathbf{P}<\mathbf{0 . 0 5})$; Ca cases, Co controls; ** Fisher's exact test used to calculate $\mathbf{P}$ value and $\mathbf{P}<\mathbf{0 . 0 5}$ considered as statistically significance 
Table 4. Summary of Multifactorial Dimensionality Reduction Analysis (MDR) for HNC Risk Prediction

\begin{tabular}{lllcl}
\hline Model & TrBA & TBA & CVC & P value \\
\hline TBS & 0.593 & 0.593 & $100 / 100$ & $<0.001$ \\
TBS, CYP1A1 & 0.592 & 0.57 & $94 / 100$ & $<0.001$ \\
TBS, TBC, CYP1A1 & 0.611 & 0.605 & $99 / 100$ & $<0.0001$ \\
TBS, TBC, ALC, CYP1A1 & 0.59 & 0.516 & $100 / 100$ & $<0.0001$ \\
\hline
\end{tabular}

TBA, testing balance accuracy; TrBA, training balance accuracy; $\mathrm{CVC}$, cross-validation consistency; TBS, tobacco smoking; TBC, tobacco chewing, ALC; Alcohol consumption; Bold values indicate best model prediction for NPC risk with highest TrBA, TBA and maximum CVC

(A)

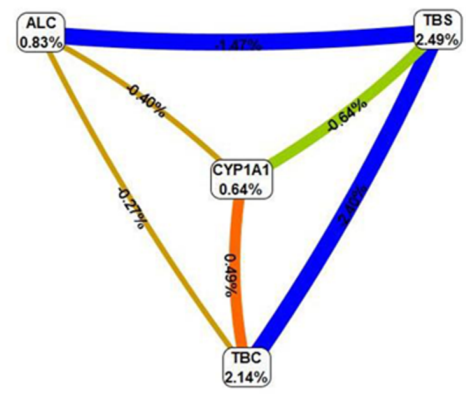

(B)

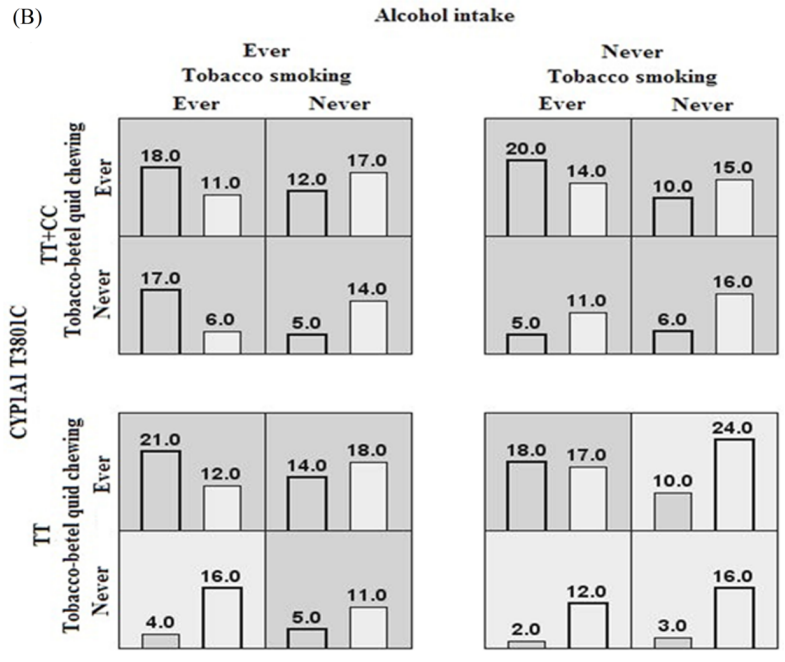

Figure 2. MDR analysis of genetic and Environmental

Factors. (A) Interaction entropy graph for gene-environment interaction and $\mathrm{HNC}$ risk. This graphical model explains the percent of the entropy in case-control removed by each factor (independent effect) and by each pair-wise combination of attributes (interaction effect). Positive percentage of entropy indicating synergistic interaction and negative values of entropy represent redundancy. The red colour indicating a high degree of synergistic interaction, orange a lesser degree whereas; gold represent midpoint; blue represents the highest level of redundancy followed by green. TBC-tobacco-betel quid chewing, TBS-tobacco smoking, and ALC-alcohol consumption; (B) Summary of the four-factor model (CYP1A1, alcohol, smoking and tobacco-betel quid chewing) in MDR analysis. The distribution of high risk (dark shading) and low risk (light shading) combinations associated with HNC risk. The percentage of patients having $\mathrm{HNC}$ was represented by left column in each box, whereas right column in each box indicated percentage of controls

HNC was observed for alcohol drinkers with smoking $(\mathrm{OR}=7.09,95 \%$ CI: $1.64-30.2 ; \mathrm{P}=0.013)$ or with tobacco- betel quid chewing (OR=7.47, 95\% CI: 1.87-29.8; $\mathrm{P}=0.010)$ and carrying $\mathrm{CC}$ genotype. However, highest risk of $\mathrm{HNC}(\mathrm{OR}=8.10,95 \% \mathrm{CI}: 2.26-29.03 ; \mathrm{P}=0.002)$ was observed for smokers with tobacco-betel quid chewing and carrying CYPIAl CC genotype.

\section{MDR analysis}

MDR analysis was used to determine the best-model gene-environment interaction for HNC risk (Table 4). The analysis suggest that smoking was the best one-factor model with CVC 100/100 (TBA=0.59; $\mathrm{P}<0.001$ ). While smoking and CYPIAl variant genotype was the best two factors model with CVC of 94/100 (TBA $=0.57 ; \mathrm{P}<0.001$ ). The best four-factors model was the combinations of smoking, tobacco-betel quid chewing, alcohol drinking and CYPlAl variant genotype with CVC of $100 / 100$ and TBA $(0.51)$ and $\mathrm{P}<0.0001$. However, the best model of all the predictive models was the three-factor model combination of smoking, tobacco-betel quid chewing, and CYPIAl variant genotype having CVC of 99/100 and highest TBA of $0.60(\mathrm{P}<0.0001)$.

\section{Interaction entropy graphs}

Interaction entropy graph was constructed using MDR results to determine synergistic or antagonistic interactions between the genetic and environmental factors in HNC risk (Figure 2). Entropy graph revealed smoking, and tobacco-betel quid chewing had highest independent effect with percentage entropy of $2.49 \%$ and $2.14 \%$, respectively. Tobacco-betel quid chewing had a synergistic interaction with CYP1Al variant $(0.64 \%)$ by removing $0.49 \%$ of entropy. Alcohol consumption ( $0.83 \%$ ) might also explain considerable entropy independently.

\section{Discussion}

In this study, we investigated the role of CYPIAl T3801C polymorphism in modulating the relationship between smoking, alcohol drinking and tobacco-betel quid chewing and HNC risk among the northeast Indian population. Our findings suggested that the variant CYP1A1 T3801C genotype, by increasing the CYP1A1 activity, modified $\mathrm{HNC}$ risk by interacting with tobacco and alcohol.

Northeast India is reported to have a very high morbidity and mortality rates of HNC (Bhattacharjee et al., 2006). Epidemiological studies from the northeast India reported tobacco smoking as one of the strongest risk factor for HNC (Yadav et al., 2010; Talukdar et al., 2013; Choudhury and Ghosh, 2014; Ghosh et al., 2014a) . Moreover, it was seen that tobacco-betel quid chewing also had a very high impact on HNC risk (Sharan et al., 2012; Mondal et al., 2013; Choudhury et al., 2014) . Tobacco in any form generates free radicals that deplete antioxidants and cause oxidative damage to DNA, proteins and lipids resulting in cancers. Similarly; alcohol consumption is linked to an increase in risk of HNC (Bektas-Kayhan et al., 2014; Ghosh et al., 2014a; Krishna et al., 2014). Recent studies have revealed mutagenic effects of alcohol on human chromosomes in vitro (Hsu et al., 1991). Our results also suggest for a strong risk of $\mathrm{HNC}$ with smoking, 
CYP1A1 T3801C Polymorphism and Tobacco and Alcohol-Associated Head and Neck Cancer Susceptibility in Northeast India alcohol drinking and tobacco-betel quid chewing (Table $1)$.

In our study, significant associations between HNC risk and CYP1A1 T3801C variant genotypes were observed (Table 2). Our study suggests an increased risk of HNC in the CYPlAl CC genotypes ( 2 fold) that could reflect the higher enzymatic activity. $C Y P 1 A l$ belongs to a superfamily of phase I xenobiotics metabolizing enzymes. These enzymes play a vital role in resisting a large variety of chemical carcinogens and environmental toxicants that are probably associated with cancer risk. Altered forms of enzymes are known to enhance enzyme activities and therefore, have been linked with increasing incidence of cancers (Sharma et al., 2013; Shukla et al., 2013), most likely due to increased susceptibilities to environmental toxins and carcinogens. Moreover, they are involved in the induction of various enzymes and proteins important in cellular function, e.g., the modulation of DNA repair genes that in turn determines individual susceptibility to cancer risks (Guo et al., 2008).

In the present study, the confounding effect of smoking on the HNC association with CYPIA1 T3801C polymorphism shows that $C Y P 1 A 1 \mathrm{CC}$ genotypes were strongly associated with risk of HNC in smokers (Table $3)$. Recent studies also observed that the risk of cancers in smokers was modulated by CYP1Al genetic variations (Guo et al., 2008; Sam et al., 2008; Liu et al., 2013; Jiang et al., 2014). However, these studies were conducted in a bi-modal manner (presence/absence) whereas our study was conducted on a dose-dependent manner. Tobacco smoke is a complex mixture of over 4,000 compounds; more than 60 of the compounds are potent carcinogens, including polycyclic aromatic hydrocarbons (PAHs), aromatic amines, N-nitroso compounds. CYPlAl is known to metabolize PAHs generated from tobacco smoke such as benzo $[\alpha]$ purene in addition to a various other carcinogenic compounds (Guengerich and Shimada, 1998; Bartsch et al., 2000). The carcinogenic substrates on exposure to $C Y P 1 A 1$ may therefore increase the potential for genomic damage (Sam et al., 2008). Studies also revealed the expression of $C Y P 1 A 1$ in the buccal mucosa, suggesting the in situ activation of tobacco carcinogens (Vondracek et al., 2001). Moreover, cigarette smoke has also been shown to up regulate $C Y P 1 A 1$ under in vitro conditions as well as in smokers (Nagaraj et al., 2006; Chi et al., 2009). Previous studies reported genetic variability altered enzyme activity and subsequently affect carcinogens detoxification in tobacco smoke, thereby increasing susceptibility to HNC risk (Soya et al., 2007; Sabitha et al., 2010; Sam et al., 2010; Tai et al., 2010; Khlifi et al., 2014). However, few studies did not find a relationship between smoking and risk of HNC among cases with the CYP1Al polymorphisms (Matthias et al., 1998; Oude Ophuis et al., 1998; Varela-Lema et al., 2008). In addition, CYP1Al TC and CC genotypes appeared to be strongly associated with risk of $\mathrm{HNC}$ in tobacco-betel quid chewers. Betel-quid chewing along with tobacco results in the exposure to nitrosamines derived from tobacco and areca or betel nut alkaloids. Betel quid chewing generate a high amount of ROS, which has been implicated in multistage carcinogenesis (Soya et al., 2007). Recent studies reported a significant association between $C Y P 1 A 1$ polymorphism and HNC among tobacco-betel quid chewers (Anantharaman et al., 2007; Hernando-Rodriguez et al., 2012; Chaudhuri et al., 2013), whereas few studies did not found any relationship between CYPIAl and chewing (Khlifi et al., 2014).

Furthermore, a strong risk modulation of HNC was observed among individual carrying the CYP1Al $\mathrm{CC}$ genotype and alcohol drinking (Table 3 ). This is the first study to report an interaction of $C Y P 1 A 1$ T3801C polymorphism and environmental factors in the development of HNC in northeast Indian population. Similar type of result, have been reported in the previous other study in oral cancers (Chatterjee et al., 2009; Chatterjee et al., 2010). On the other hand, individual carrying the CYP1A1 TC and CC genotypes and taking alcohol either with smoking or tobacco-betel quid chewing has an overall higher risk for HNC risk. Alcohol in general is not a genotoxic substance; however, its degradation results in the formation of acetaldehyde, which is carcinogenic in nature. Besides alcohol drinking suppresses the removal of nitrosamine molecules of low molecular weight released by tobacco in the liver and inhibiting several isoforms of the cytochrome P450 superfamily (Cury et al., 2012). Furthermore, there was an increase of nitrosamines to post-hepatic tissues and an increase in DNA adducts formation, which can lead to cancer development (Warnakulasuriya et al., 2005; Hashibe et al., 2007).

We include MDR analysis to study the higher order gene-environmental interaction. MDR approach identifies the combination of tobacco habits and CYP1A1 T3801C polymorphism as the best model for $\mathrm{HNC}$ risk in our study population (Table 4). Interaction entropy graph was drawn to determine synergistic or antagonistic interactions using the MDR results (Figure 2). Entropy graph, revealed tobacco habits had highest independent effect and had a synergistic interaction with CYPlAl variant genotypes. These results indicated that besides tobacco exposure, individual detoxification capacity also plays an important role in the development of HNC. Our study might have certain drawback for predicting high-order interactions due to the relatively small sample size; however, MDR approach overcomes the limitation for low sample sizes by using cross validation and permutation testing strategy.

Our study indicates that smoking, alcohol drinking and tobacco-betel quid chewing are risk factors associated with $\mathrm{HNC}$ in the northeast Indian population. In addition, it is suggested that CYP1Al T3801C polymorphism is a predisposing risk factor for HNC. The result also indicates that the risk of $\mathrm{HNC}$ associated with tobacco and alcohol is modulated by CYP 1A1 T3801C polymorphism. Highest risks of $\mathrm{HNC}$ were observed among individual consuming tobacco and alcohol and carrying the CYP1AI $\mathrm{CC}$ genotypes. These findings demonstrated importance of gene-environment interaction in prediction the susceptibility of HNC.

\section{Acknowledgements}

Authors are thankful to Department of Biotechnology, 
Government of India for providing financial support (BT/ NE/TBP/204(Med)/3/2011 and BT/Med/NE-SFC/2009). Our sincere thanks goes to Silchar Medical College and Hospital, Cachar Cancer Hospital and Research Centre biorepository, Assam; Agartala Government Medical College, Tripura and Naga Hospital Administration, Nagaland; Regional Institute of Medical Science, Manipur; Civil Hospital, Mizoram; and B. Borooah Cancer Research Institute, Assam.

\section{References}

Amtha R, Razak IA, Basuki B, et al (2014). Tobacco (kretek) smoking, betel quid chewing and risk of oral cancer in a selected Jakarta population. Asian Pac J Cancer Prev, 15, 8673-8.

Anantharaman D, Chaubal PM, Kannan S, et al (2007). Susceptibility to oral cancer by genetic polymorphisms at CYP1A1, GSTM1 and GSTT1 loci among Indians: tobacco exposure as a risk modulator. Carcinogenesis, 28, 1455-62.

Bartsch H, Nair U, Risch A, et al (2000). Genetic polymorphism of CYP genes, alone or in combination, as a risk modifier of tobacco-related cancers. Cancer Epidemiol Biomarkers Prev, 9, 3-28.

Bektas-Kayhan K, Karagoz G, Kesimli MC, et al (2014). Carcinoma of the tongue: a case-control study on etiologic factors and dental trauma. Asian Pac J Cancer Prev, 15, 2225-9.

Bhattacharjee A, Chakraborty A, Purkaystha P (2006). Prevalence of head and neck cancers in the north east-An institutional study. Indian J Otolaryngol Head Neck Surg, 58, 15-9.

Chatterjee S, Chakrabarti S, Sengupta B, et al (2009). Prevalence of CYP1A1 and GST polymorphisms in the population of northeastern India and susceptibility of oral cancer. Oncol Res, 17, 397-403.

Chatterjee S, Dhar S, Sengupta B, et al (2010). Polymorphisms of CYP1A1, GSTM1 and GSTT1 Loci as the Genetic Predispositions of Oral Cancers and Other Oral Pathologies: Tobacco and Alcohol as Risk Modifiers. Indian J Clin Biochem, 25, 260-72.

Chaudhuri SR, Mukherjee S, Paul RR, et al (2013). CYP1AI and CYP2E1 gene polymorphisms may increase susceptibility to oral submucous fibrosis among betel quid chewers of eastern India. Gene, 513, 268-71.

Chi AC, Appleton K, Henriod JB, et al (2009). Differential induction of CYP1Al and CYP1B1 by benzo[a]pyrene in oral squamous cell carcinoma cell lines and by tobacco smoking in oral mucosa. Oral Oncol, 45, 980-5.

Choudhury JH, Choudhury B, Kundu S, et al (2014). Combined effect of tobacco and DNA repair genes polymorphisms of XRCC1 and XRCC2 influence high risk of head and neck squamous cell carcinoma in northeast Indian population. Med Oncol, 31, 67.

Choudhury JH, Ghosh SK (2014). Gene-environment interaction and susceptibility in head and neck cancer patients and in their first-degree relatives: a study of Northeast Indian population. J Oral Pathol Med.

Cury NM, Russo A, Galbiatti AL, et al (2012). Polymorphisms of the CYP1A1 and CYP2E1 genes in head and neck squamous cell carcinoma risk. Mol Biol Rep, 39, 1055-63.

Ghosh SK, Mondal R (2012). Quick diagnosis of female genital tuberculosis using multiplex fast polymerase chain reaction in Southern Assam, India. Int J Gynaecol Obstet, 118, 72-3.

Ghosh SK, Singh AS, Mondal R, et al (2014a). Dysfunction of mitochondria due to environmental carcinogens in nasopharyngeal carcinoma in the ethnic group of Northeast Indian population. Tumour Biol.

Ghosh SK, Singh AS, Mondal R, et al (2014b). Dysfunction of mitochondria due to environmental carcinogens in nasopharyngeal carcinoma in the ethnic group of Northeast Indian population. Tumour Biol, 35, 6715-24.

Guengerich FP, Shimada T (1998). Activation of procarcinogens by human cytochrome P450 enzymes. Mutat Res, 400, 201-13.

Guo X, O'Brien SJ, Zeng Y, et al (2008). GSTM1 and GSTT1 gene deletions and the risk for nasopharyngeal carcinoma in Han Chinese. Cancer Epidemiol Biomarkers Prev, 17, 1760-3.

Hahn LW, Ritchie MD, Moore JH (2003). Multifactor dimensionality reduction software for detecting gene-gene and gene-environment interactions. Bioinformatics, 19, 376-82.

Hashibe M, Brennan P, Benhamou S, et al (2007). Alcohol drinking in never users of tobacco, cigarette smoking in never drinkers, and the risk of head and neck cancer: pooled analysis in the International Head and Neck Cancer Epidemiology Consortium. J Natl Cancer Inst, 99, 777-89.

Hashibe M, Brennan P, Chuang SC, et al (2009). Interaction between tobacco and alcohol use and the risk of head and neck cancer: pooled analysis in the International Head and Neck Cancer Epidemiology Consortium. Cancer Epidemiol Biomarkers Prev, 18, 541-50.

Hernando-Rodriguez M, Rey-Barja N, Marichalar-Mendia X, et al (2012). Role of cytochrome P-450 genetic polymorphisms in oral carcinogenesis. J Oral Pathol Med, 41, 1-8.

Hsu TC, Furlong C, Spitz MR (1991). Ethyl alcohol as a cocarcinogen with special reference to the aerodigestive tract: a cytogenetic study. Anticancer Res, 11, 1097-101.

Jiang XY, Chang FH, Bai TY, et al (2014). Susceptibility of lung cancer with polymorphisms of CYP1A1, GSTM1, GSTM3, GSTT1 and GSTP1 genotypes in the population of Inner Mongolia region. Asian Pac J Cancer Prev, 15, 5207-14.

Khlifi R, Chakroun A, Hamza-Chaffai A, et al (2014). Association of CYP1Al and CYP2D6 gene polymorphisms with head and neck cancer in Tunisian patients. Mol Biol Rep, 41, 2591-600.

Krishna A, Singh RK, Singh S, et al (2014). Demographic risk factors, affected anatomical sites and clinicopathological profile for oral squamous cell carcinoma in a north Indian population. Asian Pac J Cancer Prev, 15, 6755-60.

Kumar S, Muniyandi M (2015). Tobacco use and oral leukoplakia: cross-sectional study among the Gond tribe in Madhya Pradesh. Asian Pac J Cancer Prev, 16, 1515-8.

Lachenmeier DW, Przybylski MC, Rehm J (2012). Comparative risk assessment of carcinogens in alcoholic beverages using the margin of exposure approach. Int J Cancer, 131, 9951003.

Lavender NA, Benford ML, VanCleave TT, et al (2009). Examination of polymorphic glutathione S-transferase (GST) genes, tobacco smoking and prostate cancer risk among men of African descent: a case-control study. BMC Cancer, 9, 397.

Liu C, Jiang Z, Deng QX, et al (2014). Meta-analysis of association studies of $C Y P 1 A l$ genetic polymorphisms with digestive tract cancer susceptibility in Chinese. Asian Pac J Cancer Prev, 15, 4689-95.

Liu L, Wu G, Xue F, et al (2013). Functional CYP1Al genetic variants, alone and in combination with smoking, contribute to development of head and neck cancers. Eur J Cancer, 49, 2143-51.

Lu Y,Zhang XL, Xie L, et al (2014). Lack of association between CYP1Al polymorphisms and risk of bladder cancer: a meta- 
CYP1A1 T3801C Polymorphism and Tobacco and Alcohol-Associated Head and Neck Cancer Susceptibility in Northeast India

analysis. Asian Pac J Cancer Prev, 15, 4071-7.

Masood N, Yasmin A, Kayani MA (2014). Genetic variations and head and neck cancer risks. Mol Biol Rep, 41, 2667-70.

Matthias C, Bockmuhl U, Jahnke V, et al (1998). Polymorphism in cytochrome P450 CYP2D6, CYP1A1, CYP2E1 and glutathione S-transferase, GSTM1, GSTM3, GSTT1 and susceptibility to tobacco-related cancers: studies in upper aerodigestive tract cancers. Pharmacogenetics, 8, 91-100.

Mondal R, Ghosh SK (2013). Accumulation of mutations over the complete mitochondrial genome in tobacco-related oral cancer from northeast India. Mitochondrial DNA, 24, 432-9.

Mondal R, Ghosh SK, Choudhury JH, et al (2013). Mitochondrial DNA copy number and risk of oral cancer: a report from Northeast India. PLoS One, 8, 57771.

Nagaraj NS, Beckers S, Mensah JK, et al (2006). Cigarette smoke condensate induces cytochromes $\mathrm{P} 450$ and aldo-keto reductases in oral cancer cells. Toxicol Lett, 165, 182-94.

$\mathrm{Ng}$ DP, Tan KW, Zhao B, et al (2005). CYP1Al polymorphisms and risk of lung cancer in non-smoking Chinese women: influence of environmental tobacco smoke exposure and GSTM1/T1 genetic variation. Cancer Causes Control, 16, 399-405.

Olshan AF, Weissler MC, Watson MA, et al (2000). GSTM1, GSTT1, GSTP1, CYP1A1, and NAT1 polymorphisms, tobacco use, and the risk of head and neck cancer. Cancer Epidemiol Biomarkers Prev, 9, 185-91.

Oude Ophuis MB, van Lieshout EM, Roelofs HM, et al (1998). Glutathione S-transferase M1 and T1 and cytochrome $\mathrm{P} 4501 \mathrm{~A} 1$ polymorphisms in relation to the risk for benign and malignant head and neck lesions. Cancer, 82, 936-43.

Risch A, Ramroth H, Raedts V, et al (2003). Laryngeal cancer risk in Caucasians is associated with alcohol and tobacco consumption but not modified by genetic polymorphisms in class I alcohol dehydrogenases ADH1B and ADH1C, and glutathione-S-transferases GSTM1 and GSTT1. Pharmacogenetics, 13, 225-30.

Sabitha K, Reddy MV, Jamil K (2010). Smoking related risk involved in individuals carrying genetic variants of CYP1A1 gene in head and neck cancer. Cancer Epidemiol, 34, 587-92.

Sam SS, Thomas V, Reddy KS, et al (2010). Gene-environment interactions associated with CYP1Al MspI and GST polymorphisms and the risk of upper aerodigestive tract cancers in an Indian population. J Cancer Res Clin Oncol, 136, 945-51.

Sam SS, Thomas V, Reddy SK, et al (2008). CYPlAl polymorphisms and the risk of upper aerodigestive tract cancers in an Indian population. Head Neck, 30, 1566-74.

Sharan RN, Mehrotra R, Choudhury Y, et al (2012). Association of betel nut with carcinogenesis: revisit with a clinical perspective. PLoS One, 7, 42759.

Sharma A, Das BC, Sehgal A, et al (2013). GSTM1 and GSTT1 polymorphism and susceptibility to esophageal cancer in high- and low-risk regions of India. Tumour Biol, 34, 3249-57.

Shukla RK, Tilak AR, Kumar C, et al (2013). Associations of CYP1A1, GSTM1 and GSTT1 polymorphisms with lung cancer susceptibility in a Northern Indian population. Asian Pac J Cancer Prev, 14, 3345-9.

Siegel R, Ma J, Zou Z, et al (2014). Cancer statistics, 2014. CA Cancer J Clin, 64, 9-29.

Soya SS, Vinod T, Reddy KS, et al (2007). Genetic polymorphisms of glutathione-S-transferase genes (GSTM1, GSTT1 and GSTP1) and upper aerodigestive tract cancer risk among smokers, tobacco chewers and alcoholics in an Indian population. Eur J Cancer, 43, 2698-706.

Tai J, Yang M, Ni X, et al (2010). Genetic polymorphisms in cytochrome $\mathrm{P} 450$ genes are associated with an increased risk of squamous cell carcinoma of the larynx and hypopharynx in a Chinese population. Cancer Genet Cytogenet, 196, 76-82.

Talukdar FR, Ghosh SK, Laskar RS, et al (2013). Epigenetic, genetic and environmental interactions in esophageal squamous cell carcinoma from northeast India. PLoS One, $\mathbf{8 , 6 0 9 9 6 . ~}$

Varela-Lema L, Taioli E, Ruano-Ravina A, et al (2008). Metaanalysis and pooled analysis of GSTM1 and CYP1A1 polymorphisms and oral and pharyngeal cancers: a HuGEGSEC review. Genet Med, 10, 369-84.

Vondracek M, Xi Z, Larsson P, et al (2001). Cytochrome P450 expression and related metabolism in human buccal mucosa. Carcinogenesis, 22, 481-8.

Warnakulasuriya S, Sutherland G, Scully C (2005). Tobacco, oral cancer, and treatment of dependence. Oral Oncol, 41, 244-60.

Wu X, Zhao H, Suk R, et al (2004). Genetic susceptibility to tobacco-related cancer. Oncogene, 23, 6500-23.

Yadav DS, Devi TR, Ihsan R, et al (2010). Polymorphisms of glutathione-S-transferase genes and the risk of aerodigestive tract cancers in the Northeast Indian population. Genet Test Mol Biomarkers, 14, 715-23.

Yu X, Liu J, Zhu H, et al (2014). Synergistic association of DNA repair relevant gene polymorphisms with the risk of coronary artery disease in northeastern Han Chinese. Thromb Res, 133, 229-34 